\title{
Análise numérica de vigas de rolamento de aço sem contenção lateral entre apoios
}

\author{
Luiz Rafael dos Santos Leite ${ }^{1}$ e Maximiliano Malite ${ }^{2 *}$ \\ ${ }^{1}$ Eng. Civil, Agência Reguladora de Serviços Públicos Delegados de Transporte \\ do Estado de São Paulo - ARTESP/SP. \\ Irafaelsantosl@gmail.com \\ 2 Professor do Departamento de Engenharia de Estruturas da EESC-USP \\ Av. Trabalhador São-Carlense, 400 - São Carlos, SP \\ mamalite@sc.usp.br
}

\section{Numerical analysis of crane runaway girders of steel without lateral bracing between supports.}

\begin{abstract}
Resumo
As vigas de rolamento de pontes rolantes são elementos estruturais sujeitos à ação conjunta dos momentos fletor e de torção, sendo este provocado pela excentricidade da força vertical da roda e pelo impacto lateral durante a operação da ponte. Na fase de projeto, a combinação dos momentos de flexão e torção geralmente é reduzida ao caso de flexão atuando nos dois eixos principais da seção transversal da viga e, de maneira menos usual, a combinação dos momentos é analisada segundo a teoria da flexo-torção. Este trabalho apresenta a comparação entre as tensões longitudinais calculadas pelos modelos teóricos de barras e pela análise numérica, por meio do Método dos Elementos Finitos (MEF), para o caso da viga de rolamento de aço sem contenção lateral entre apoios. A comparação mostrou divergência entre os resultados e que os modelos de barra podem levar a situações tanto contra como a favor da segurança.
\end{abstract}

Palavras-chave: Vigas de aço, vigas de rolamento, pontes rolantes, flexo-torção.

\begin{abstract}
The crane runway girders are structural elements subjected to a combined action of flexural and torsional moments, being this last one caused by the eccentricity of the wheel's vertical force and by the side thrust during the crane operation. In the design phase, the combination of flexural and torsion moments is generally reduced to flexural moments acting over the principal axes of transverse section of the girder and, in a less usual way, the combination of moments is analyzed by the flexural-torsional theory. This research presents a comparison between the longitudinal stresses calculated by the classical bars models and by the numerical analysis through the Finite Element Method (FEM), in case of the crane runway girder of steel without lateral bracing between support points. The comparison showed a divergence between the results and that the classical bars models can lead to situations of high safety or against safety.
\end{abstract}

Keywords: Steel girders, runway girder, cranes, flexural-torsional. 


\section{Introdução}

As vigas de rolamento são estruturas de suporte para pontes rolantes, sendo estas utilizadas para içamento e movimentação de cargas. As solicitações verticais impostas às vigas incluem o seu peso próprio, o peso da ponte, o peso dos dispositivos para operação e a carga içada. Forças horizontais provocadas pela operação da ponte também atuam sobre a viga e, eventualmente, momentos de torção.

O momento torçor tem basicamente duas causas: a força vertical da roda da ponte, quando excêntrica ao eixo de simetria vertical da seção da viga, sendo a excentricidade causada geralmente por imprecisões na montagem do caminho de rolamento ou pelo seu desalinhamento sobre a viga. A outra causa é a inevitável excentricidade da força horizontal transversal à viga, denominada de impacto lateral, a qual atua fora do centro de cisalhamento da seção. Uma vez que a excentricidade da força vertical, a intensidade das forças e o sentido do impacto lateral podem variar durante a operação, o momento torçor pode ser máximo, quando as parcelas se somam, ou mínimo, quando se reduzem. O presente trabalho traz uma análise, via método dos elementos finitos, da influência da torção sobre a viga de rolamento, examinando a contribuição desta solicitação nas tensões longitudinais e, em seguida, comparando estes valores com as tensões calculadas pelos modelos clássicos de barras, a fim de verificar se os mesmos representam adequadamente o comportamento da viga de rolamento.

O perfil I é o mais utilizado para vigas de rolamento e, a depender do vão da viga e da capacidade nominal da ponte rolante, será executado um sistema de travamento lateral para garantir a estabilidade global da estrutura. A tabela 1 mostra relações práticas entre capacidade nominal da ponte, vãos da viga de rolamento e travamentos utilizados. Foram abordadas vigas de rolamento com seção monossimétrica, vão livre entre 6 e 7 metros e que suportam pontes com capacidade nominal máxima de 250 kN. Nessas condições, o travamento lateral é feito apenas na região dos apoios formando o chamado vínculo de garfo, o qual é responsável por transferir o impacto lateral aos pilares do edifício. Quando comparadas às vigas de seção duplamente simétrica, as vigas de seção monossimétrica apresentam maior estabilidade lateral se a mesa maior resulta comprimida. 
Tabela 1 - Travamento lateral da viga de rolamento (BELLEI, 2000. Adaptado).

\begin{tabular}{lll}
\hline $\begin{array}{c}\text { Capacidade nominal } \\
\text { da ponte rolante }\end{array}$ & $\begin{array}{c}\text { Vão da viga de } \\
\text { rolamento }\end{array}$ & $\begin{array}{c}\text { Seção da viga e sistema de } \\
\text { travamento lateral }\end{array}$ \\
\hline Até $50 \mathrm{kN}$ & Até 6 metros & $\begin{array}{l}\text { Perfil I com dupla simetria sem } \\
\text { travamento lateral }\end{array}$ \\
\hline De $50 \mathrm{kN}$ até $250 \mathrm{kN}$ & Até 7 metros & $\begin{array}{l}\text { Perfil I monossimétrico sem } \\
\text { travamento lateral }\end{array}$ \\
\hline De $50 \mathrm{kN}$ até $250 \mathrm{kN}$ & Até 13 metros & $\begin{array}{l}\text { Perfil I com contenção na mesa } \\
\text { superior }\end{array}$ \\
\hline Acima de $250 \mathrm{kN}$ & ---- & $\begin{array}{l}\text { Perfil I ou caixão com contenção } \\
\text { na mesa superior e inferior }\end{array}$ \\
\hline
\end{tabular}

\section{Projeto e cálculo da tensão longitudinal da viga de rolamento}

O dimensionamento da viga de rolamento envolve algumas simplificações no problema estrutural, dentre elas, a "transformação" das solicitações dinâmicas da ponte em solicitações estáticas equivalentes por meio dos coeficientes de impacto. Além disso, o valor do impacto lateral, o qual é tomado como uma parcela da força vertical da roda, sofre grande variação em função do código normativo adotado em seu cálculo.

Por exemplo, a norma norte-americana Minimum Design Loads for Building and Other Structures, elaborada pela American Society of Civil Engineers (ASCE/SEI 7-10), determina que o impacto lateral, independentemente do mecanismo de operação e utilidade da ponte, seja igual a $20 \%$ da soma da capacidade nominal da ponte, peso da talha e do trole. Por sua vez, o Technical Report no.13 - Guide for Design and Construction of Mill Buildings, elaborado pela Association of Iron and Steel Engineers ${ }^{1}$ (AISE n13:2003), é mais criterioso, especificando a intensidade do impacto lateral em função de características da ponte. Embora os relatórios técnicos da AISE não tenham valor normativo e sejam fontes auxiliares, por vezes, o seu detalhamento é mais abrangente, sendo, por isso, preferidos para projetos.

Já a norma brasileira para estruturas em aço, a ABNT NBR 8800:2008 - Projeto de estruturas de aço e de estruturas mistas de aço e concreto de edifícios (ABNT NBR 8800:2008) segue a linha da AISE n13, especificando o valor do impacto lateral em

\footnotetext{
${ }^{1}$ Em 2004 a Association of Iron and Steel Engineers (AISE) e a Iron and Steel Society (ISS) uniram-se dando origem a Association for Iron \& Steel Technology (AIST).
} 
função das características e utilidade da ponte rolante. A tabela 2, adaptada de FISHER ${ }^{2}$ (2004) para incluir a norma brasileira, mostra que para a mesma ponte rolante encontram-se três valores diferentes para o impacto lateral e cada valor pode levar a um dimensionamento e solução estrutural diferente.

Tabela 2 - Valor total do impacto lateral - exemplo (FISHER, 2004. Adaptado).

$$
\text { Características da ponte rolante: }
$$

Edifício industrial com ponte rolante de capacidade nominal: $1.000 \mathrm{kN}(\mathrm{A})$

Peso do trole, incluindo talha e demais dispositivos de içamento: $300 \mathrm{kN}$ (B)

Peso da composição ponte, trole e dispositivos de içamento: $785 \mathrm{kN}$ (C)

\begin{tabular}{ccc}
\hline Documento de referência & $\begin{array}{c}\text { Considerar o } \\
\text { maior valor entre: }\end{array}$ & Impacto lateral (kN) \\
\hline ASCE/SEI 7-10 & $0,20(\mathrm{~A}+\mathrm{B})$ & 260 \\
\hline & $0,40(\mathrm{~A})$ & 400 \\
AISE n13:2003 & $0,20(\mathrm{~A}+\mathrm{B})$ & 260 \\
& $0,10(\mathrm{~A}+\mathrm{C})$ & 178,5 \\
\hline \multirow{2}{*}{ ABNT NBR 8800:2008 } & $0,10(\mathrm{~A}+\mathrm{B}) \times 2$ & 260 \\
(comandada por cabine) & $0,05(\mathrm{~A}+\mathrm{C}) \times 2$ & 178,5 \\
& $0,15(\mathrm{~A}) \times 2$ & 300 \\
\hline
\end{tabular}

A aceleração e a frenagem da ponte produzem, ainda, forças horizontais longitudinais na direção do eixo da viga de rolamento, porém elas são utilizadas no dimensionamento do para-choque da ponte e do sistema de contraventamento vertical do edifício, os quais não foram alvos deste estudo.

O momento torçor pode ser substituído por um par de forças atuando nas mesas superior e inferior do perfil, simplificação conhecida como analogia de flexão. Esta alteração modifica o problema da torção para flexão atuando nos planos da mesa e desconsidera a contribuição do momento de torção distribuído provocado pela carga do trilho excêntrico à viga de rolamento. A analogia de flexão, por sua simplicidade, é mais utilizada em projetos de vigas de rolamento e é apresentada na figura 1.

Pode-se utilizar também a teoria da flexo-torção para considerar a contribuição da torção na tensão longitudinal. Determinando o bimomento $B$, que é um esforço autoequilibrado na seção da viga, e o giro $\phi$ da seção calcula-se o acréscimo na tensão longitudinal devido à torção (tensão normal de flexo-torção). Esta abordagem é mais

\footnotetext{
${ }^{2}$ FISHER utilizou a ASCE 7 de 2002. A revisão ASCE/SEI 7-10 de 2010 apresenta os mesmos critérios para determinação do impacto lateral, portanto, a comparação ainda é válida.
} 
complexa, pois depende da solução de equações diferenciais e do conhecimento das condições de contorno de cada problema.

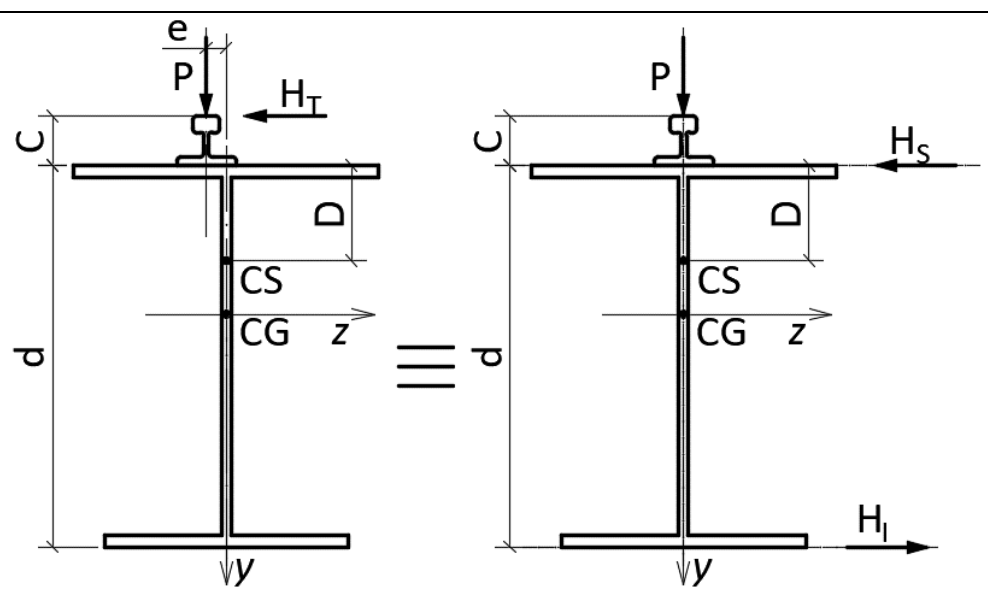
a) Problema Real
b) Simplificação de projeto

P: força vertical da roda da ponte, já majorada pelo coeficiente de impacto;

$\mathrm{H}_{\mathrm{T}}$ : impacto lateral da ponte;

e: excentricidade da força vertical;

d: altura total do perfil;

C: altura do trilho ou caminho de rolamento (se houver);

CG: centro geométrico da seção;

CS: centro de cisalhamento da seção;

D: distância entre o CS da seção e o topo da mesa superior;

$\mathrm{H}_{\mathrm{s}}$ : força aplicada na mesa superior, conforme equação (1);

$\mathrm{H}_{1}$ : força aplicada na mesa inferior, conforme equação (2).

Figura 1 - Atuação das forças da ponte sobre a viga de rolamento - Analogia de flexão.

$\mathrm{H}_{\mathrm{S}}=\frac{\mathrm{Pe}+\mathrm{H}_{\mathrm{T}}(\mathrm{d}+\mathrm{C})}{\mathrm{d}}=\mathrm{H}_{\mathrm{T}}+\frac{\mathrm{Pe}+\mathrm{H}_{\mathrm{T}} \mathrm{C}}{\mathrm{d}}$

$\mathrm{H}_{\mathrm{l}}=\frac{\mathrm{Pe}+\mathrm{H}_{\mathrm{T}} \mathrm{C}}{\mathrm{d}}$

A figura 2 mostra o problema da viga de rolamento equacionado para determinação do bimomento e giro da seção, sendo o trem-tipo da ponte posicionado na situação de máximo momento fletor. São omitidos na figura o diagrama de momento fletor e o carregamento distribuído, pois não são necessários neste cálculo. A equação diferencial do giro para o problema, após integração, resulta na expressão (3), na qual os coeficientes $\alpha_{i}$ são constantes de integração que dependem das condições de contorno, G é o módulo de elasticidade transversal (77.000 MPa), J é o momento de inércia à 
torção da seção e r é o comprimento de comparação, definido na expressão (4) como função de $G$, de J, da constante de empenamento da seção $C_{w}$, também denominada de integral de empenamento, e do módulo de elasticidade longitudinal E (200.000 MPa).

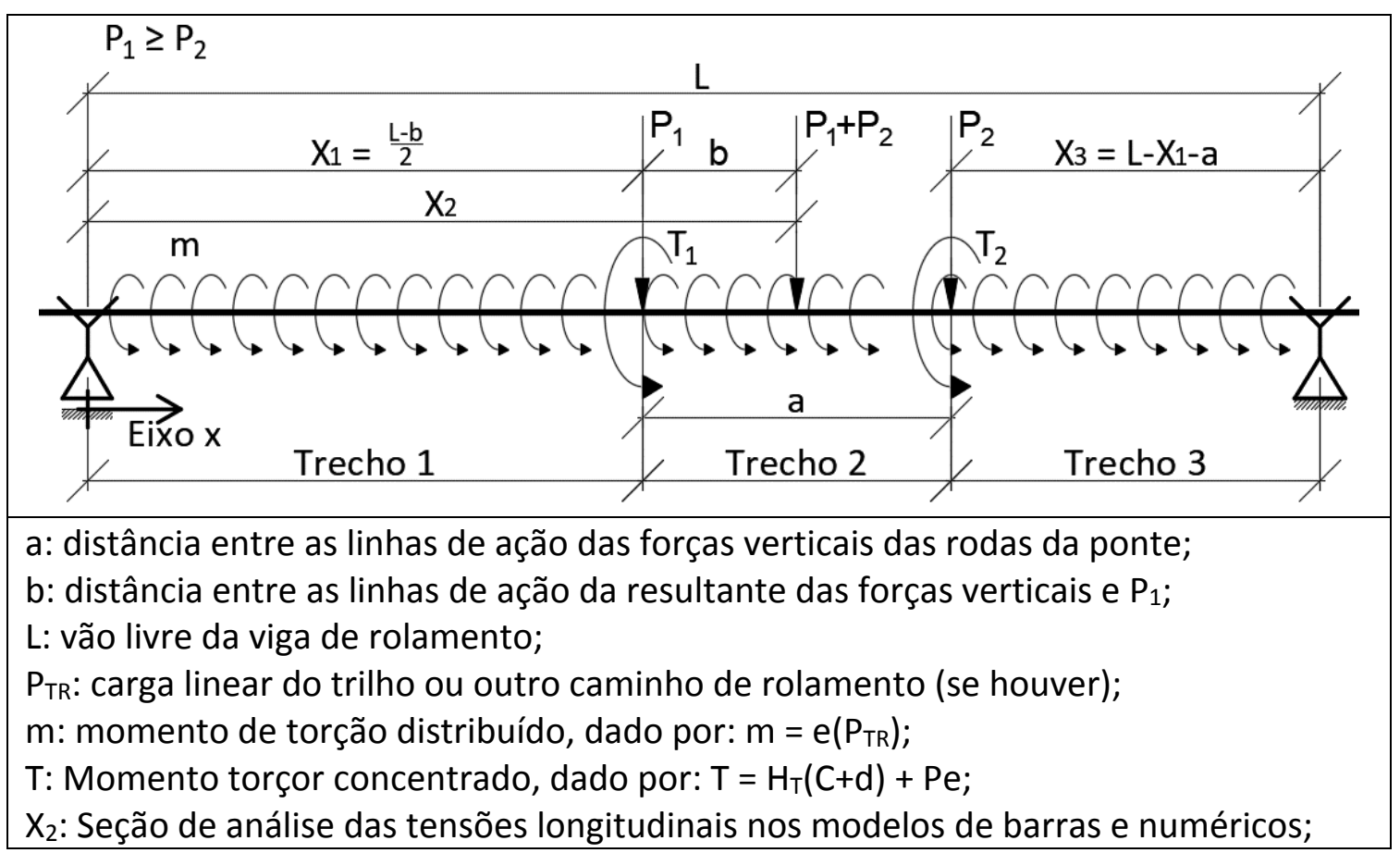

Figura 2 - Solicitações sobre a viga de rolamento - equacionamento para flexo-torção.

$$
\begin{aligned}
& \phi_{i}=\alpha_{i 1} \operatorname{senh}\left(\frac{x}{r}\right)+\alpha_{i 2} \cosh \left(\frac{x}{r}\right)+\alpha_{i 3} x+\alpha_{i 4}+\frac{m x^{2}}{2 G J} \quad i=1,2,3 \\
& r=\sqrt{\frac{E C_{w}}{G J}}
\end{aligned}
$$

A tensão longitudinal $\sigma_{x}$ devido a flexo-torção é calculada em cada seção de interesse conforme a expressão (5), na qual $\omega$ é a área setorial principal da seção. Por último, vale lembrar que as propriedades geométricas da seção $C_{w}$, J e $\omega$ são constantes para o mesmo perfil, uma vez que neste trabalho são consideradas apenas barras prismáticas.

$$
\sigma_{x}=E \omega \phi_{i}^{\prime \prime}=\frac{B_{i} \omega}{C_{w}} \quad i=1,2,3
$$

\section{Modelo Numérico de Elementos Finitos}

Com o objetivo de avaliar se os modelos de barras apresentados na seção 2 representam adequadamente as tensões longitudinais da viga, um modelo tridimensional de 
elementos finitos reproduzindo uma viga biapoiada com vínculos de garfo na região dos apoios foi construído no programa ANSYS (2010). No caso real, em geral são empregadas chapas com baixa rigidez à flexão conectando a mesa superior do perfil I ao pilar. Ao invés de introduzir a chapa de ligação no modelo numérico, poderiam ser impostas restrições de deslocamento diretamente à mesa superior, porém, este método provoca rigidez excessiva na extremidade da mesa, afastando o modelo do problema real. Enrijecedores transversais de alma foram utilizados apenas na região dos apoios.

Dois tipos de elementos finitos, ambos naturais da biblioteca do ANSYS, foram utilizados para construir o modelo. Utilizou-se o elemento de casca SHELL 181 para a viga e enrijecedores, pois ele é adequado para seções delgadas, além de permitir a análise não linear geométrica com a plastificação da seção. Já para a chapa de ligação foi escolhido o elemento de barra LINK 8, também capaz de deformar plasticamente. A escolha de um elemento tipo LINK (barra) ao invés de elemento BEAM (viga) levou em consideração a baixa rigidez à flexão da chapa de ligação. O refinamento da malha é maior na mesa superior do perfil, uma vez que ela é mais importante para análise. A diferença entre o refinamento na mesa superior e inferior gera uma zona de transição na malha do enrijecedor, contudo, essa transição não produziu problemas ou erros na análise.

O trilho não foi construído no modelo e a contribuição de sua carga foi aplicada sobre a mesa superior por meio de pressão distribuída. A carga concentrada da roda da ponte rolante também foi aplicada em forma de pressão sobre sua área de espraiamento, adotando-se o ângulo de espraiamento de $45^{\circ}$, valor usual em projetos. 0 impacto lateral, atuante no boleto do trilho, foi transportado para a mesa superior como um conjunto de forças horizontais e verticais, de maneira a simular os pontos de fixação do trilho na viga de rolamento, como ilustra a figura 3.

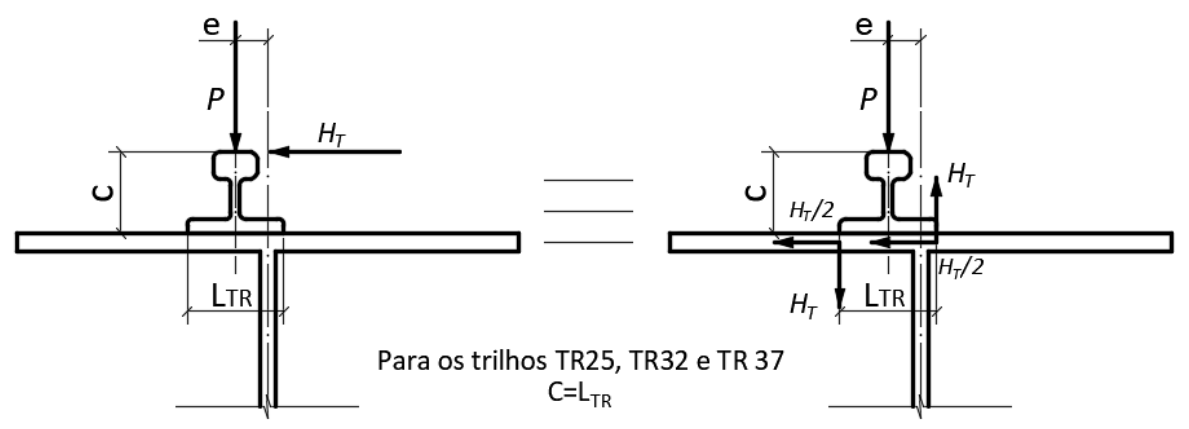

Figura 3-Consideração do impacto lateral no modelo numérico. 
A figura 4.a mostra a perspectiva geral do modelo numérico, enquanto a figura 4.b mostra o detalhe da transição da malha no enrijecedor e da vinculação externa da viga. A figura 4.c, por sua vez, traz as solicitações aplicadas na viga. Nela pode-se notar a faixa de carregamento correspondente ao trilho e as áreas de aplicação das cargas das rodas e, finalmente, a figura 4.d ilustra a aplicação do impacto lateral na mesa superior.

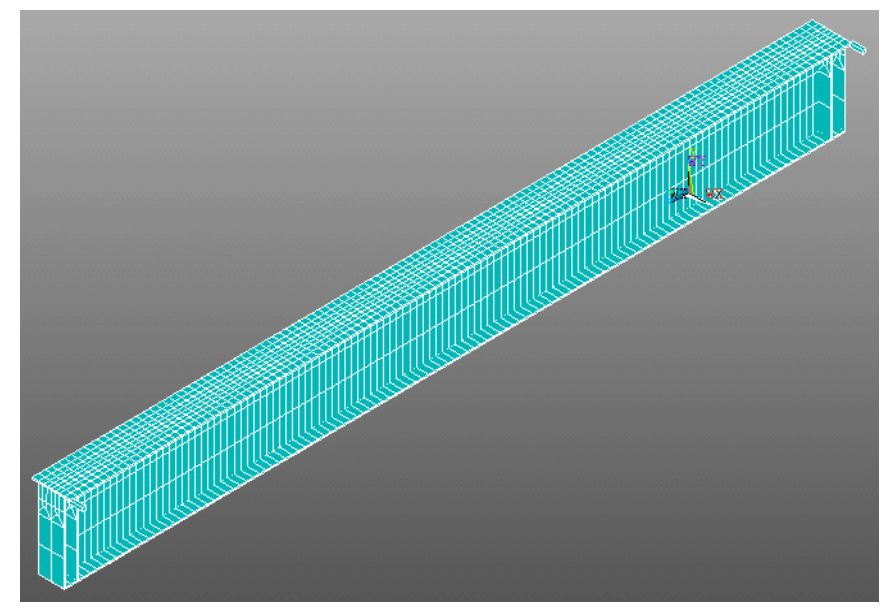

a) Viga de rolamento em elementos finitos.

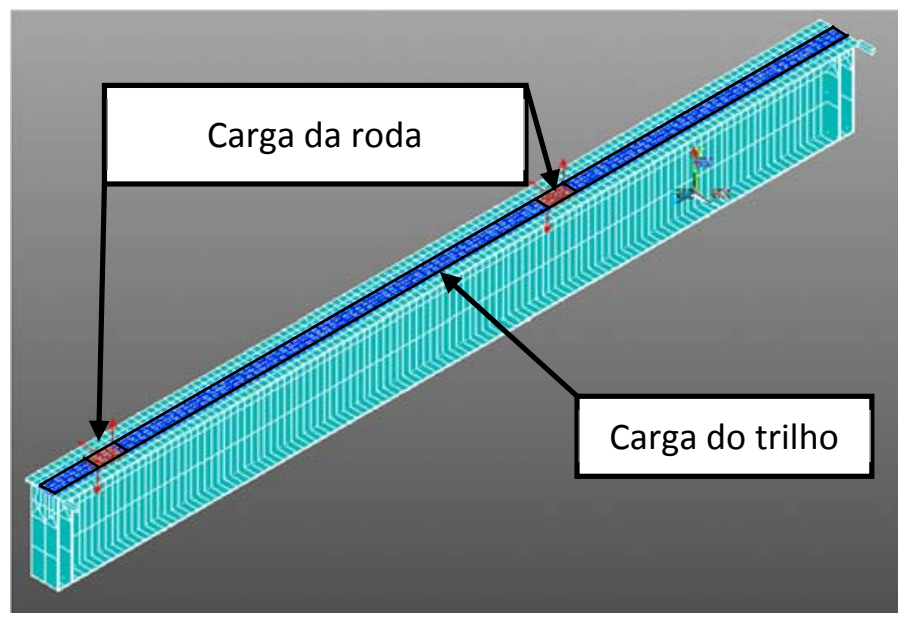

c) Ações no modelo numérico

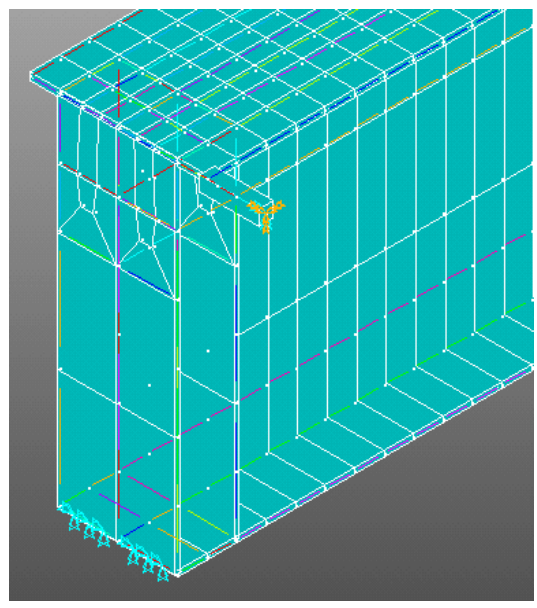

b) Detalhe região do apoio.

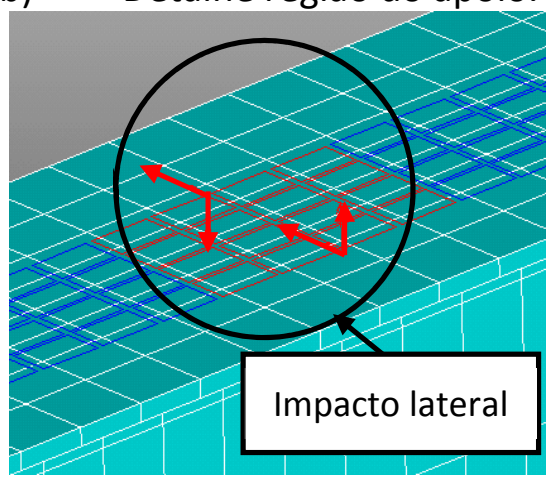

d) Impacto lateral

transportado para a mesa.

Figura 4 - Modelo numérico.

As propriedades constitutivas do aço foram introduzidas com o diagrama tensão $\mathrm{x}$ deformação trilinear apresentado na figura 5. Ele considera o critério de plastificação de von Mises, possibilitando simular o comportamento elasto-plástico isótropo do material. Já a não linearidade geométrica do problema foi considerada pela formulação Lagrangeana e para realizar as iterações optou-se pelo método completo NewtonRaphson (Full N-R), o qual inclui a atualização da matriz de rigidez em todas as iterações nas quais houve incremento de carga da ponte rolante. 


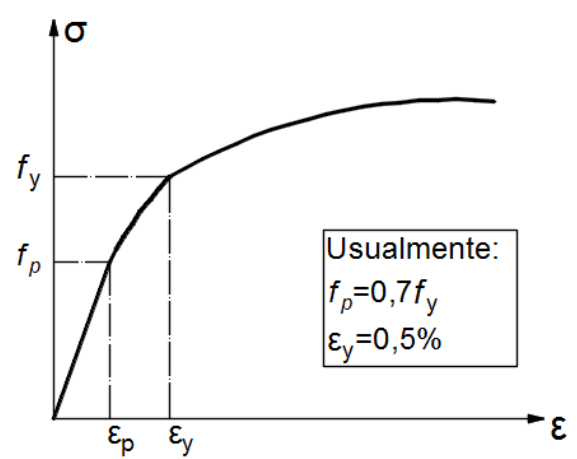

(a)

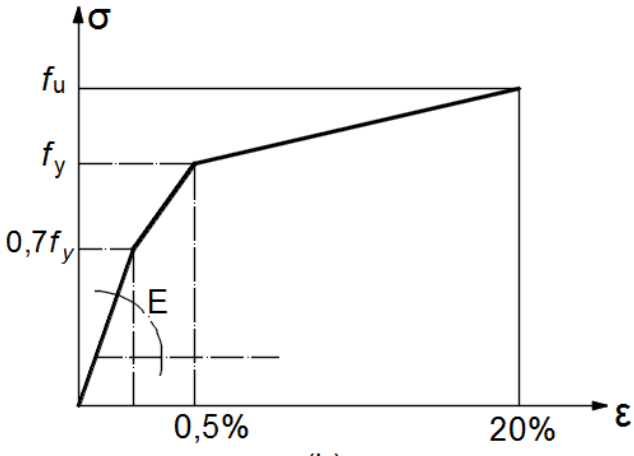

(b)

Figura 5 - (a) Diagrama tensão $x$ deformação com comportamento elasto-plástico do aço e (b) diagrama trilinear utilizado no modelo numérico.

\section{Comparação entre modelos teóricos de barras e modelo numérico}

A tensão longitudinal, pela teoria da flexo-torção, tem sinal em função do bimomento e da área setorial principal, sendo os eixos coordenados, a convenção de sentidos positivos para bimomento, momento torçor concentrado, momento torçor distribuído e giro da seção mostrados na figura 6.

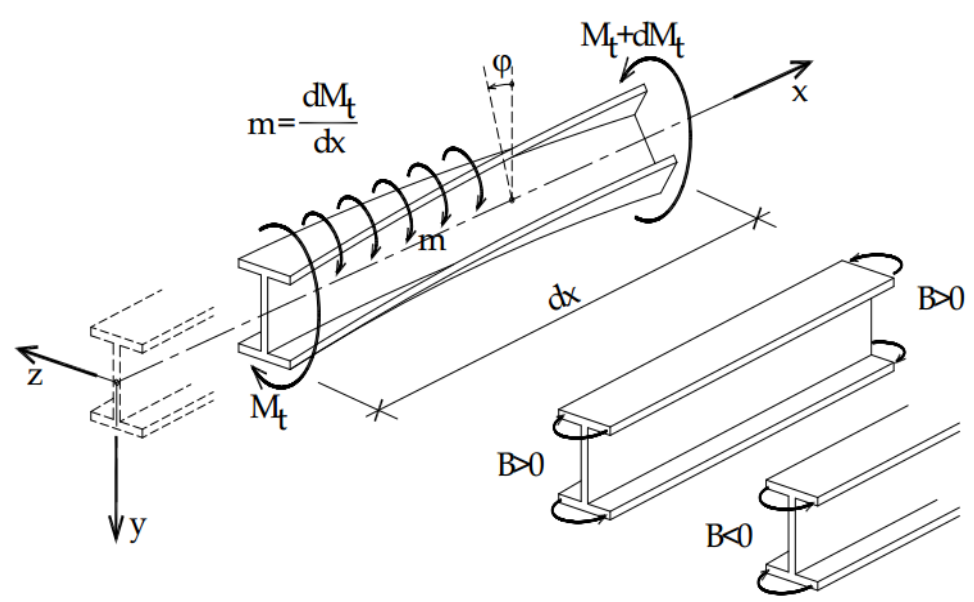

Figura 6 - Sentidos positivos para B, $M_{t}$, m e $\phi$ (Mori; Neto,2009).

O diagrama da área setorial principal para o perfil I monossimétrico é indicado na figura

7 e nela aparecem também os pontos de controle para análise da tensão. Se bimomento e área setorial são positivos, considerando que a flexão em torno de z provoca na mesa superior tensões de compressão e na inferior tração, a variação de $\sigma_{x}$ no modelo de barra ocorre da seguinte maneira: redução da tensão de compressão em $\mathrm{p} 1_{\mathrm{fs}}$ e aumento 
em $\mathrm{p} 5_{\mathrm{fs}}$; redução da tensão de tração em $\mathrm{p} 1_{\mathrm{fi}}$ e aumento em $\mathrm{p} 3_{\mathrm{fi}}$. A área setorial principal é nula no plano da alma e não há variação de $\sigma_{\mathrm{x}}$. As variáveis geométricas que aparecem na figura 7 são as dimensões do perfil, sendo $b_{\mathrm{fi}}$ a largura da mesa inferior, $b_{\mathrm{fs}}$ a largura da mesa superior, $t_{f i}$ a espessura da mesa inferior, $t_{f s}$ a espessura da mesa superior e $t_{w}$ a espessura da alma.

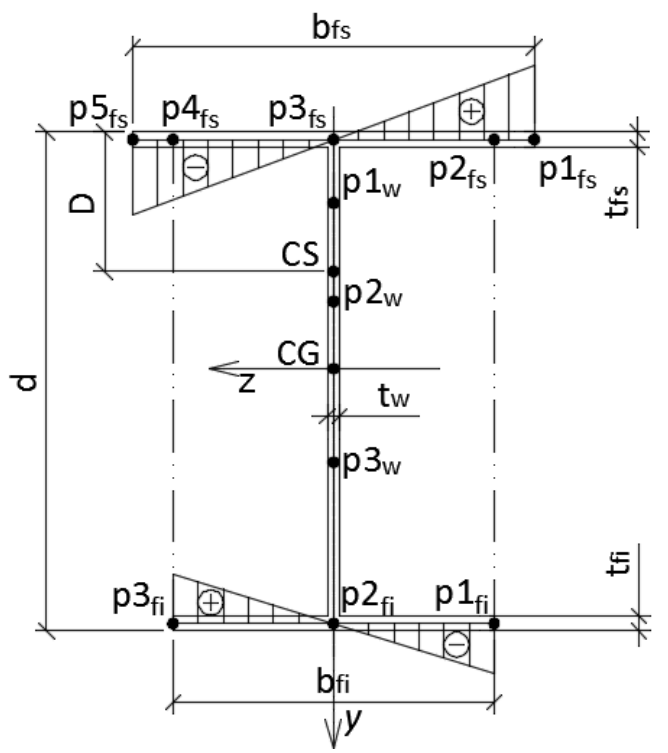

Figura 7 - Sentido positivo para $\omega$.

$\mathrm{Na}$ analogia de flexão, para o momento fletor $M_{y}$, em torno do eixo $y$, adotou-se como positiva a orientação mostrada na figura 8 , porque desta forma a variação da tensão longitudinal nas mesas é correspondente à teoria da flexo-torção. As mesas, geralmente, são tratadas de maneira independente e submetidas à flexão no próprio plano, portanto as tensões são calculadas dividindose o momento fletor $M_{y s}$ ou $M_{y i}$ pelo respectivo módulo de resistência elástico.

Alguns parâmetros do problema estrutural foram fixados, uma vez que é ampla a combinação entre vãos de viga, perfil de viga e trens-tipos de pontes rolantes. Para a viga analisada foi escolhido o perfil soldado monossimétrico PSM 550x75 (Figura 9).

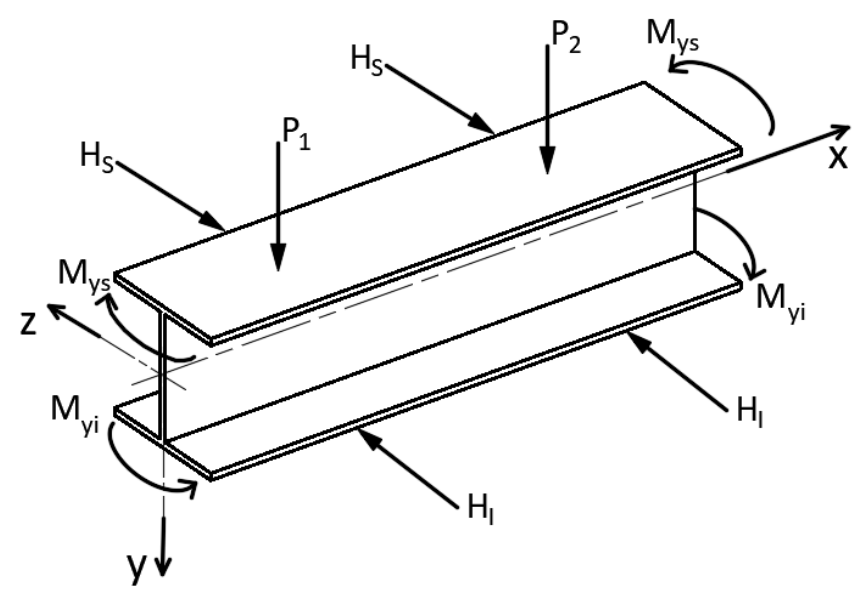

Figura 8 - Sentido positivo para $\mathrm{M}_{\mathrm{y}}$.

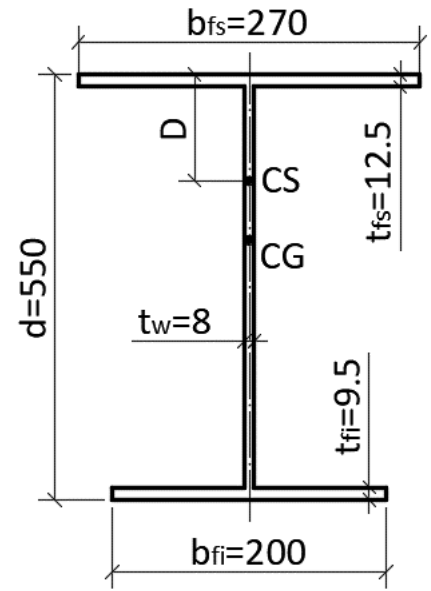

Figura 9 - Perfil PSM 550x75. 
A fadiga não foi considerada nesse trabalho, mas tomou-se como referência a limitação de tensão no item k.2.2 da ABNT NBR 8800:2008, cujo valor máximo, considerando combinação frequente de fadiga, é 0,66 $f_{y}$ para tensões normais e 0,40 $f_{y}$ para tensões de cisalhamento, assegurando o trabalho do material em regime elástico.

A majoração da força vertical da roda foi de $10 \%$ (operação por controle pendente) e o valor do impacto lateral tomado como igual a $10 \%$ da força vertical majorada, sendo que para a análise não foi considerado o peso próprio da ponte e demais dispositivos de içamento. O material usado em todas as análises foi o aço ASTM A36, cujos valores nominais de resistência ao escoamento, $f_{\mathrm{y}}$, e à ruptura, $f_{\mathrm{u}}$, são de $250 \mathrm{MPa}$ e $400 \mathrm{MPa}$, respectivamente. Logo, a tensão longitudinal limite para combinação frequente de fadiga resulta em $165 \mathrm{MPa}(0,66 f \mathrm{y})$. O vão da viga foi de 6,3 metros e o trem-tipo da ponte é mostrado na figura 10.

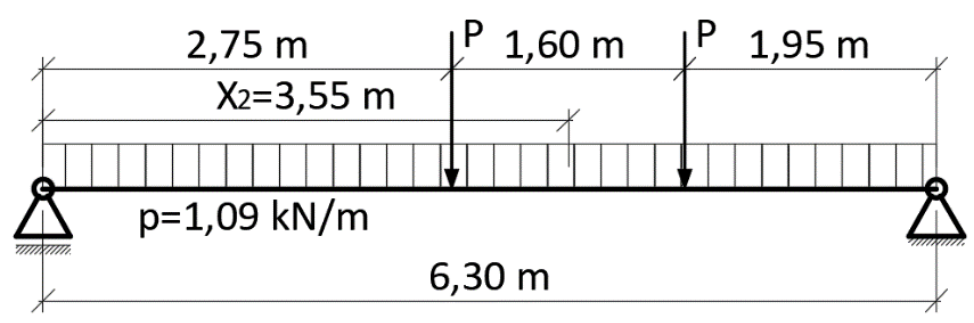

Figura 10 - Trem-tipo na posição crítica correspondente ao máximo momento fletor.

A comparação das tensões longitudinais entre os modelos de barras e o numérico foi realizada na seção $X_{2}$ (figura 10), pois nela há menor ou nenhuma influência dos efeitos localizados das solicitações. A tabela 3 contém o resumo do dimensionamento em relação a flexão, segundo critério da ABNT NBR 8800:2008, de cada um dos carregamentos analisados. O valor inicial de $P, 44 \mathrm{kN}$, está bem abaixo do limite prático utilizado para vigas de rolamento sem contenção lateral e o valor máximo apresentado, 110 kN, leva a tensões na mesa inferior superiores ao limite de proporcionalidade, porém, o momento resistente de cálculo, $M_{R d}$, é superior ao momento solicitante de cálculo $M_{S d}$, uma vez que ainda não foram considerados os efeitos da torção sobre $\sigma_{\mathrm{x}}$. Os valores de tensões longitudinais na tabela 3 estão um pouco a baixo do esperado em função do aumento linear do carregamento, pois o incremento das solicitações não foi aplicado na carga distribuída, apenas na força aplicada pela roda da ponte. 
Tabela 3 - Resumo do dimensionamento à flexão.

\begin{tabular}{cccc}
\hline $\begin{array}{c}\text { Força vertical } \\
\text { majorada }(k N)\end{array}$ & $\begin{array}{c}\sigma_{x} \text { para combinação } \\
\text { frequente }(\mathrm{MPa})\end{array}$ & $\mathrm{M} s d_{\text {sd }}(\mathrm{kN} . \mathrm{m})$ & $\mathrm{M}_{\text {Rd }}(\mathrm{kN} . \mathrm{m})$ \\
\hline $\mathrm{P}=44$ & 75 & 173 & 434 \\
$\mathrm{P}=66$ & 111 & 252 & 434 \\
$\mathrm{P}=88$ & 147 & 332 & 434 \\
$\mathrm{P}=110$ & 183 & 411 & 434 \\
\hline
\end{tabular}

O valor máximo da excentricidade vertical, referenciado nas tolerâncias executivas da AISE n13:2003, é o maior valor entre $3 / 4$ de $t_{w}$ e 6,35 mm. Neste estudo adotou-se o limite superior de $10 \mathrm{~mm}$ para investigar situações desfavoráveis não previstas em projeto. Também no intuito de verificar o caso mais prejudicial, as parcelas dos momento de torção, devido ao impacto lateral e excentricidade vertical do carregamento, foram combinadas de maneira a maximizar a solicitação sobre a viga de rolamento.

Na figura 11.a, em linhas cheias, aparecem as máximas tensões de compressão no modelo numérico devido à torção causada apenas pela excentricidade do trilho e, em linhas tracejadas, a tensão causada pela adição do impacto lateral ao momento torçor, maximizando seu valor. De maneira análoga, a figura 11.b mostra a tensão de tração na mesa inferior. Primeiramente, nota-se que o limite de proporcionalidade, linha horizontal contínua nas figuras 11.a e 11.b, é superado com o aumento da intensidade da força vertical e excentricidade do trilho, desrespeitando a condição imposta pela norma brasileira. Rompendo este limite, outras condições normativas perdem validade, o que pode levar a erros de projeto e, em situações mais graves, a ruína da estrutura.

Outra análise mostra que a máxima tensão de compressão na mesa superior sofre considerável acréscimo com a introdução do impacto lateral e, na presença do mesmo, o aumento da excentricidade provoca redução da máxima tensão de compressão. Esse resultado é divergente do esperado pelos modelos de barra, seja pela teoria da flexotorção ou analogia de flexão, uma vez que em um dos extremos da mesa deveria ocorrer acréscimo na compressão conforme cresce a intensidade do momento torçor. A tensão de tração, por sua vez, é sempre crescente e a diferença provocada pela adição do impacto lateral ao momento torçor é menos acentuada. 


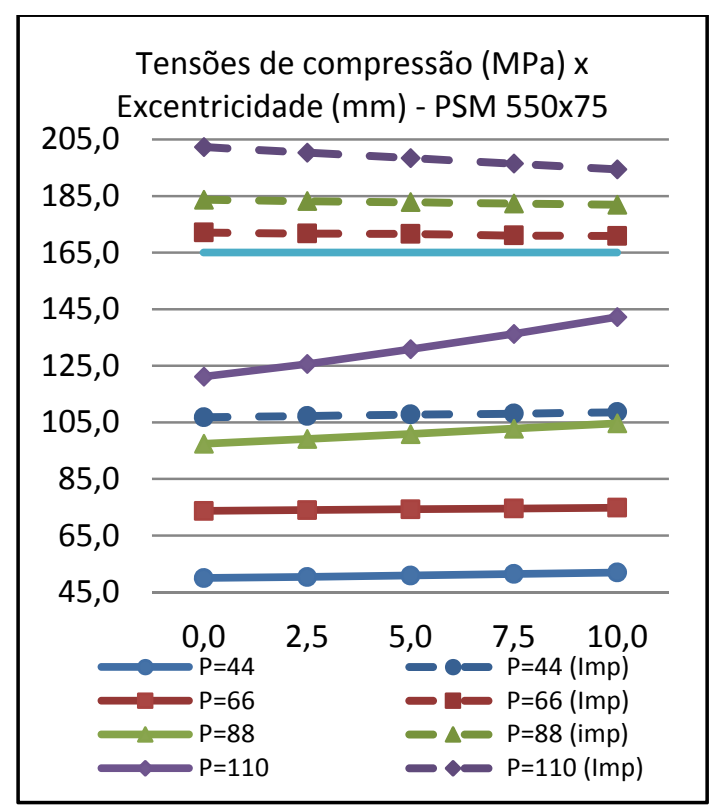

(a)

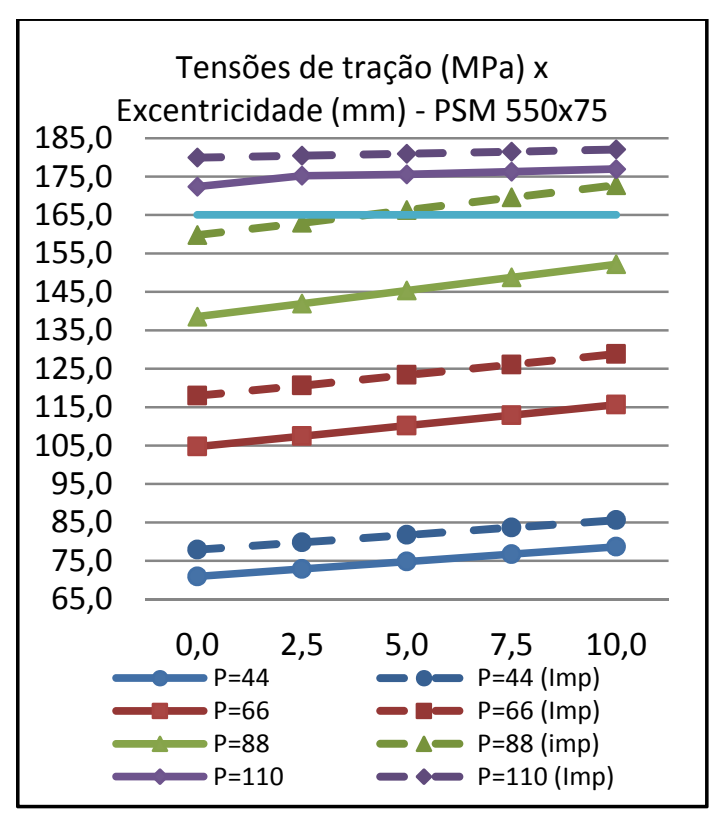

(b)

Figura 11 - (a) Tensões longitudinais de compressão com e sem impacto lateral e (b)

tensões longitudinais de tração com e sem impacto lateral - modelo numérico.

A divergência dos resultados na presença do impacto lateral pode ser explicada, em parte, pelo efeito alavanca que ele provoca sobre a mesa superior, o qual é de difícil análise por meio da sobreposição de efeitos e não é abordado nos modelos de barra. Vale ressaltar, ainda, que o mesmo efeito gera sobre a mesa superior tensões longitudinais localizadas de tração com elevada intensidade, podendo superar a tração na mesa inferior. A figura 12 apresenta essas tensões localizadas e as linhas tracejadas mostram a posição indeslocada da viga.
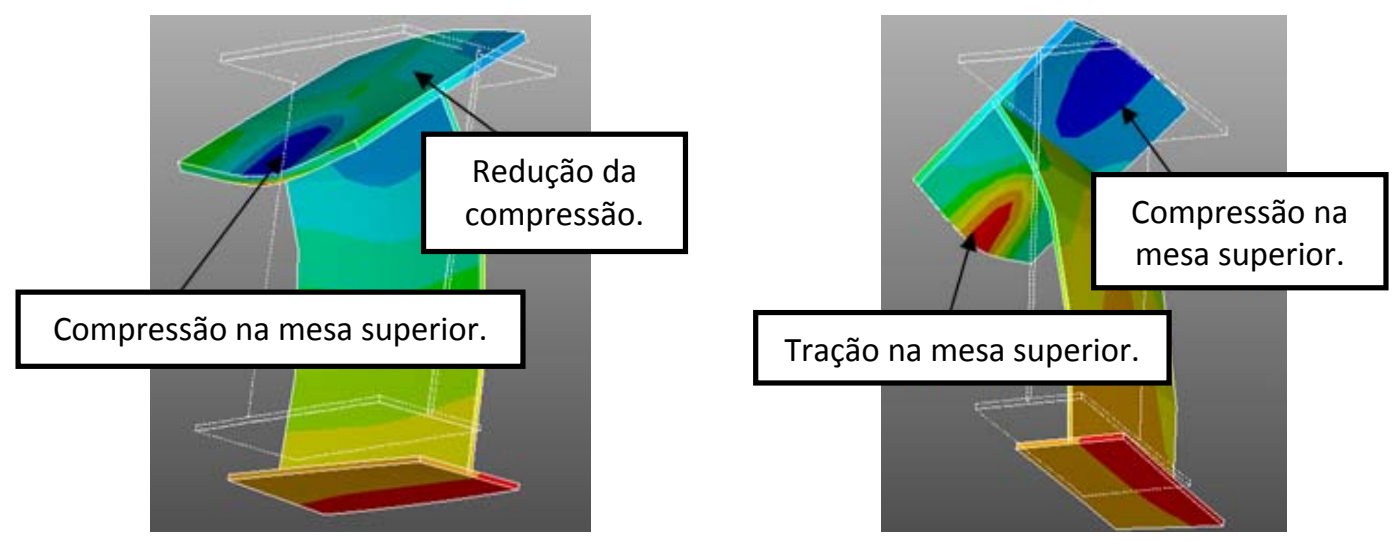

Figura 12 - Tensões longitudinais na mesa superior próximas à região de aplicação do impacto lateral. 
Uma vez que o impacto lateral atuante no modelo numérico leva a resultados qualitativos diferentes do esperado, na comparação quantitativa de $\sigma_{x}$ entre modelos de barra e numérico, considerou-se apenas o efeito da excentricidade do trilho.

Analisando as tensões nos pontos extremos da mesa superior, $\mathrm{p} 1_{\mathrm{fs}}$ e $\mathrm{p} 5_{\mathrm{fs}}$, nos quais deveriam ocorrer redução e aumento da tensão de compressão, respectivamente, o modelo numérico apresentou uma inversão, ou seja, houve aumento da compressão no ponto $\mathrm{p} 1_{\mathrm{fs}}$ e redução em $\mathrm{p} 5_{\mathrm{fs}}$ (figuras 13.a e 13.b). Essa inversão não ocorreu na mesa inferior e a variação da tensão de tração é maior no modelo da flexo-torção do que a resposta do modelo numérico (figuras 14.a e 14.b). Nas figuras 13 e 14 as linhas cheias são referentes ao modelo numérico e as tracejadas à teoria da flexo-torção.

A inversão do sentido do bimomento na mesa superior é influenciada pela intensidade da força da roda, como pode ser observado na tabela 4, que mostra as tensões longitudinais provocadas pela força de $44 \mathrm{kN}$ e $66 \mathrm{kN}$ no ponto de p1 $1_{\mathrm{fs}}$. Embora os valores da flexo-torção e numéricos sejam divergentes, para a menor força o bimomento no MEF tem mesmo sentido que o esperado da flexo-torção. Já para a força de 66 kN percebe-se o início da inversão do sentido do bimomento.

Fixando-se o valor de $66 \mathrm{kN}$ para a força vertical da roda e aumentando a espessura da mesa superior, ou seja, tornando-a mais rígida, o sentido do bimomento no modelo numérico volta a condizer com o esperado do modelo flexo-torção, demonstrando que a espessura da mesa também influencia a inversão do bimomento. No caso particular analisado, espessuras iguais ou superiores a $16 \mathrm{~mm}$ levaram ao sentido esperado do bimomento, como pode-se observar na tabela 5.

Além da inversão do sentido do bimomento, percebeu-se também que o equilíbrio da seção no modelo numérico não é possível considerando apenas a variação de tensões nas mesas. O acréscimo de compressão de um lado da mesa superior não é anulado pela redução do lado oposto, sendo o equilíbrio mantido devido à contribuição da alma, fato contrário aos modelos de barras, os quais indicam que no plano da alma do perfil não ocorre variação da tensão longitudinal. Apesar de terem sido apresentados nos gráficos e tabelas apenas os valores da flexo-torção para comparação, as mesmas divergências nos resultados foram encontradas entre MEF e analogia de flexão. 


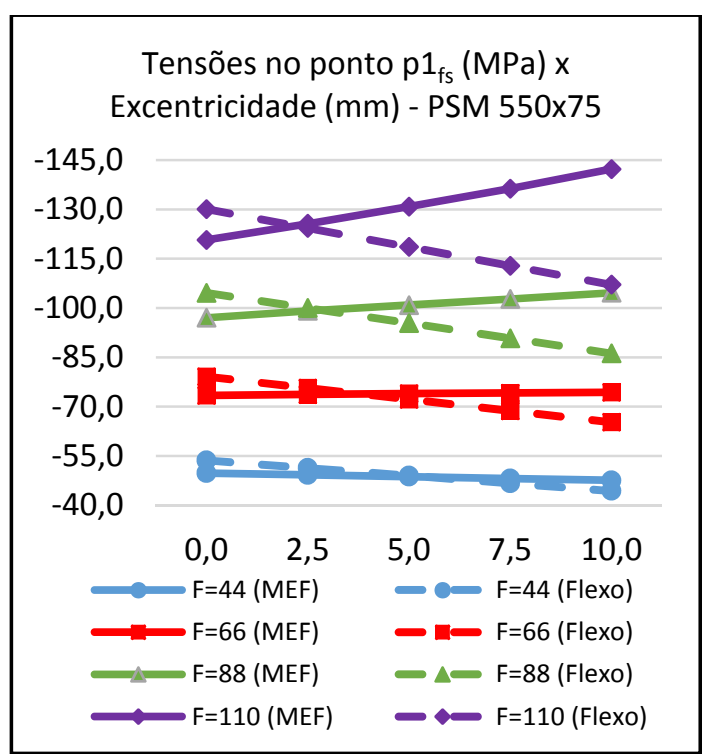

(a)

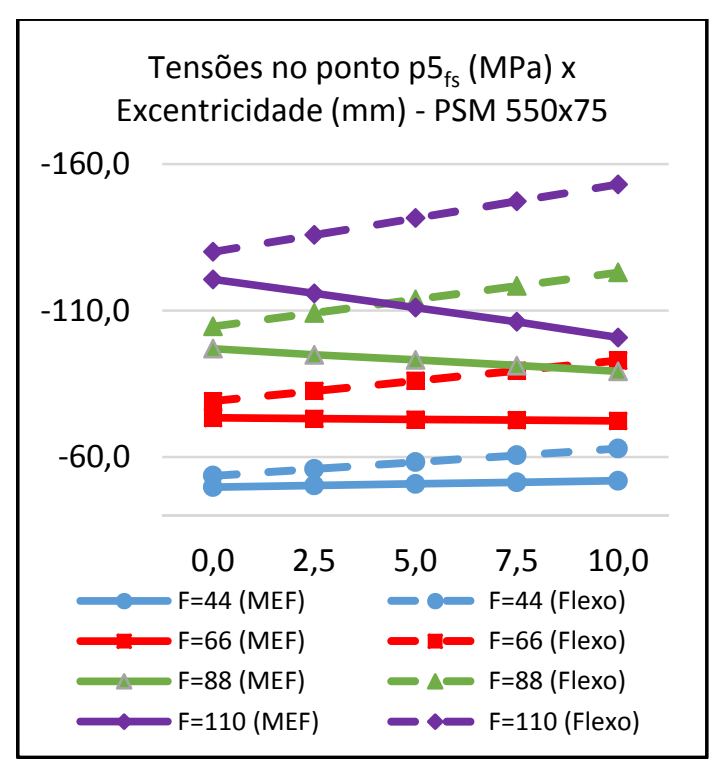

(b)

Figura 13 - Tensões longitudinais de compressão no modelo numérico e teoria da flexo-torção em $p 1_{f s}(a)$ e $p 5_{f s}(b)$.

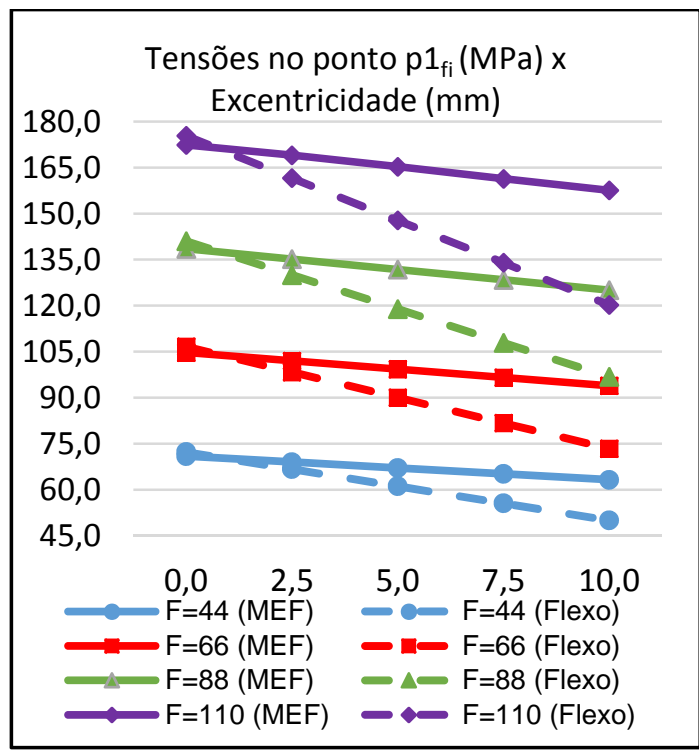

(a)

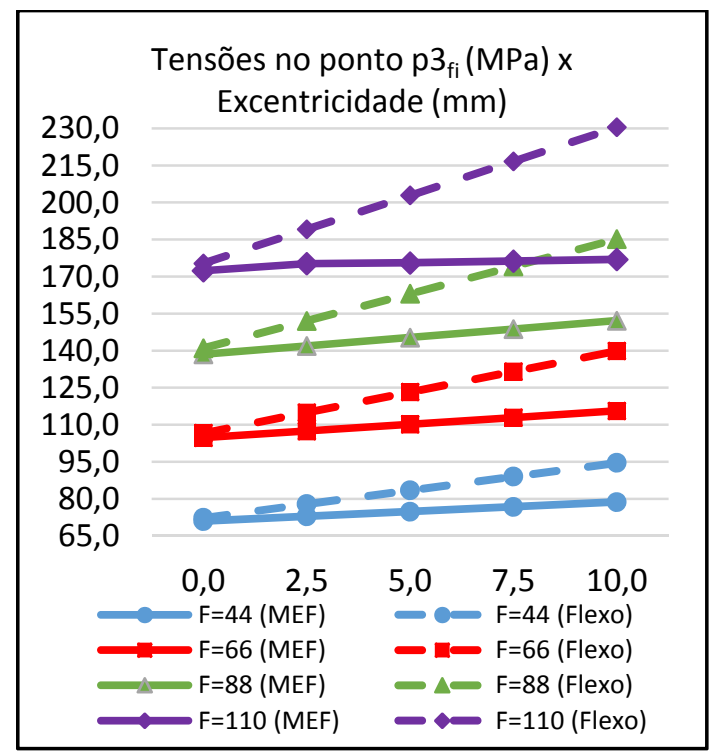

(b)

Figura 14 - Tensões longitudinais de tração no modelo numérico e teoria da flexo-

torção em $p 1_{f i}(a)$ e $p 3_{f i}(b)$.

Tabela 4 - Tensão de compressão na mesa superior - PSM 550x75

\begin{tabular}{ccccc}
\hline \multirow{2}{*}{ Excentricidade $(\mathrm{mm})$} & \multicolumn{4}{c}{$\sigma_{\mathrm{x}} \mathrm{p} 1_{\mathrm{fs}}(\mathrm{MPa})$} \\
\cline { 2 - 5 } & $44 \mathrm{kN}(\mathrm{MEF})$ & $44 \mathrm{kN}($ Flexo) & $66 \mathrm{kN}(\mathrm{MEF})$ & $66 \mathrm{kN}$ (Flexo) \\
\hline 0,0 & $-49,75$ & $-53,65$ & $-73,40$ & $-79,13$ \\
2,5 & $-49,19$ & $-51,33$ & $-73,68$ & $-75,67$ \\
5,0 & $-48,65$ & $-49,00$ & $-73,96$ & $-72,20$ \\
7,5 & $-48,11$ & $-46,68$ & $-74,11$ & $-68,74$ \\
10,0 & $-47,56$ & $-44,36$ & $-74,34$ & $-65,27$ \\
\hline
\end{tabular}


Tabela 5 - Tensão de compressão na mesa superior, $\mathrm{P}=66 \mathrm{kN}$ e $\mathrm{t}_{\mathrm{fs}}$ variável.

\begin{tabular}{ccccc}
\hline Espessura $(\mathrm{mm})$ & $12,5(\mathrm{MEF})$ & $16(\mathrm{MEF})$ & $20(\mathrm{MEF})$ & $25,4(\mathrm{MEF})$ \\
\hline Excentricidade $(\mathrm{mm})$ & \multicolumn{5}{c}{$\sigma_{\mathrm{x}}$ em p1 $1_{\mathrm{fs}}$ MEF $(\mathrm{MPa})$} \\
\hline 0,0 & $-73,40$ & $-60,41$ & $-49,97$ & $-40,14$ \\
2,5 & $-73,68$ & $-60,21$ & $-49,66$ & $-39,88$ \\
5,0 & $-73,96$ & $-60,01$ & $-49,36$ & $-39,61$ \\
7,5 & $-74,11$ & $-59,83$ & $-49,06$ & $-39,36$ \\
10,0 & $-74,34$ & $-59,63$ & $-48,75$ & $-39,09$ \\
\hline
\end{tabular}

\section{Dimensionamento e comparações de tensões longitudinais.}

Nesta seção é apresentado um exemplo de dimensionamento de viga de rolamento segundo os critérios da ABNT NBR 8800:2008. O vão da viga, trem-tipo da ponte rolante e a seção de comparação entre as tensões longitudinais dos modelos de barra e numéricos estão indicados na figura 15. O perfil analisado é o PSM 600x90, cujas dimensões são mostradas na figura 16 e, na mesma figura, L.N.P. é a linha neutra plástica e L.N.E. a linha neutra elástica da seção.

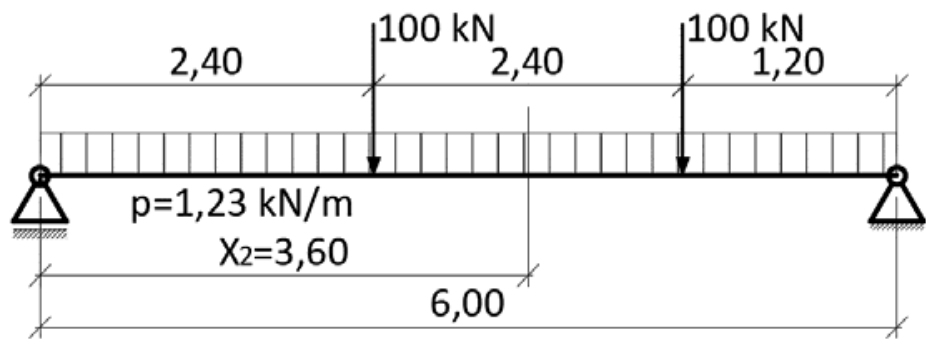

Figura 15 - Trem-tipo da ponte rolante para exemplo de dimensionamento.

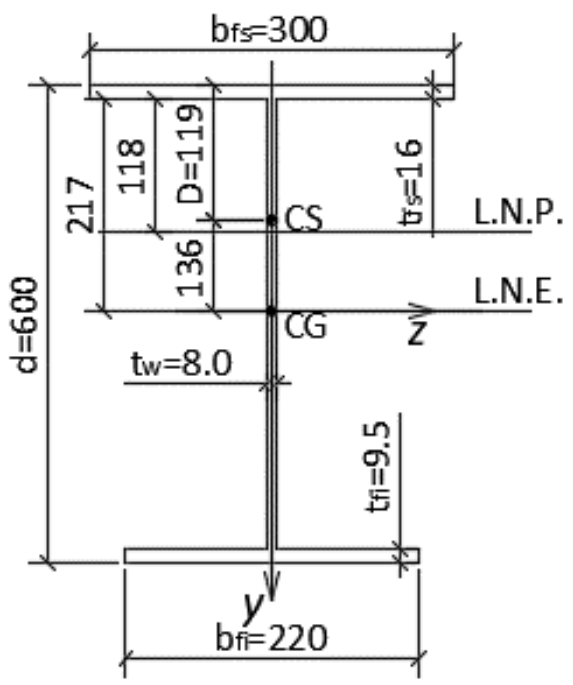

Figura 16 - Perfil PSM 600x90.
O carregamento proposto respeita as condições normativas limites de proporcionalidade das tensões longitudinal e cisalhante para combinações frequentes de fadiga, além de atender as características geométricas da seção monossimétrica, escoamento local da alma, estado limite último de enrugamento da alma e flambagem lateral da alma (anexo G, itens 5.7.3, 5.7.4 e 5.7.5 da ABNT NBR 8800:2008, respectivamente) 
O dimensionamento à flexão mostra que o perfil escolhido é adequado para resistir ao carregamento, uma vez que o momento resistente de cálculo é igual a 559 kN.m, enquanto o momento solicitante de cálculo é de $295 \mathrm{kN} . \mathrm{m}$, ou seja, este representa $52,7 \%$ do momento resistente. A verificação da força cortante é igualmente satisfeita, pois a máxima força cortante solicitante de cálculo é de $245 \mathrm{kN}$ e a resistente de cálculo é de $636 \mathrm{kN}$.

Ao carregamento proposto foram adicionados o impacto lateral de $10 \mathrm{kN}$ e a excentricidade acidental de $4 \mathrm{~mm}$ do trilho, sendo adotado o TR 37. As tensões longitudinais de cálculo na seção média entre as rodas da ponte, $\mathrm{X}_{2}$, foram determinadas por meio da analogia de flexão e também pela teoria da flexo-torção para comparação com os resultados do modelo numérico.

Pela analogia de flexão, as forças horizontais atuando sobre as mesas superior e inferior são 12,7 kN e 2,7 kN, respectivamente. Na teoria da flexo-torção é possível considerar o momento distribuído, sendo este igual a $1,82 \times 10^{-3} \mathrm{kN} . \mathrm{m} / \mathrm{m}$. O momento torçor concentrado na flexo-torção, devido ao impacto lateral, é igual a 4,22 kN.m e, por fim, a área setorial principal nos pontos extremos das mesas superior e inferior são $1,6713 \times 10^{-2} \mathrm{~m}^{2}$ e $5,2341 \times 10^{-2} \mathrm{~m}^{2}$, respectivamente.

Após a majoração das ações permanentes e variáveis, foram determinadas as tensões longitudinais de cálculo, $\sigma_{x S d}$, as quais são apresentadas na tabela 6 . Vale salientar que neste exemplo também ocorreu inversão do sentido do bimomento na mesa superior. A tabela 7 traz os coeficientes de integração para as condições da figura 15, já tendo sido considerada a majoração das solicitações variáveis e permanentes.

A análise dos resultados mostrou que, pela analogia de flexão, a tensão de compressão na mesa superior é superestimada, superando a tensão no modelo numérico em $25,3 \%$, enquanto pela teoria da flexo-torção a tensão é subestimada, sendo $40,1 \%$ inferior à obtida via MEF. Para as tensões de tração, ambos os modelos de barra levam a resultados superestimados. Na analogia de flexão a tensão estimada é $36,9 \%$ superior e, na teoria da flexo-torção, 51,5\%. 
Tabela 6 - Tensões longitudinais solicitantes de cálculo $\sigma_{\text {xSd }}(\mathrm{MPa})$

\begin{tabular}{cccc}
\hline & Analogia de flexão & Flexo-torção & MEF \\
\hline Máxima compressão & 223 & 127 & 178 \\
Máxima tração & 230 & 256 & 169 \\
\hline
\end{tabular}

Tabela 7 - Coeficientes de integração para trem-tipo da figura 15.

\begin{tabular}{ccc}
\hline Trecho 1 & Trecho 2 & Trecho 3 \\
\hline$\alpha_{11}=7,7885$ & $\alpha_{21}=-9,9341$ & $\alpha_{31}=-41,4816$ \\
\hline$\alpha_{12}=-0,0195$ & $\alpha_{22}=11,0501$ & $\alpha_{32}=39,3994$ \\
\hline$\alpha_{13}=-36,3355$ & $\alpha_{23}=9,0106$ & $\alpha_{33}=54,3568$ \\
\hline$\alpha_{14}=0,2098$ & $\alpha_{24}=-108,6210$ & $\alpha_{34}=-326,2825$ \\
\hline
\end{tabular}

\section{Conclusões}

As análises realizadas via método de elementos finitos permitiram verificar algumas das hipóteses utilizadas nos modelos de barras, as quais servem também de base para o dimensionamento da viga de rolamento. Primeiramente, foram constatadas divergências entre os valores das tensões longitudinais dos modelos de barra, seja da teoria da flexo-torção ou da analogia de flexão, quando comparados com as tensões no modelo numérico. Essas diferenças são decorrentes, em parte, do efeito alavanca que o impacto lateral provoca sobre a viga, levando a deslocamentos excessivos e invalidando a hipótese simplificadora de pequenos deslocamentos dos modelos de barras.

Os resultados mostraram também uma inversão do sentido do bimomento na mesa superior, alterando os valores de tensão nos pontos onde era esperado aumento e redução de compressão. A intensidade da força vertical e rigidez da mesa superior podem influenciar nesta inversão. Além disso, constatou-se que no modelo numérico ocorre variação da tensão longitudinal na alma do perfil devido ao momento torçor, o que não está de acordo com nenhum dos modelos de barras.

Outro ponto que os modelos de barras têm dificuldades de representar é a diferença entre rigidez das mesas superior e inferior do perfil I e, de maneira adequada, atribuir a cada uma a respectiva parcela da capacidade portante da viga. Essa dificuldade levou a divergências na determinação das tensões solicitantes e conclui-se que isto pode acarretar em diferentes dimensionamentos a favor ou contra a segurança. 


\section{Referências bibliográficas}

AMERICAN INSTITUTE OF STEEL CONSTRUCTION. (2010). ANSI/AISC 360-10: Specification for Structural Steel Buildings. Chicago, IL, 2010.

AMERICAN SOCIETY OF CIVIL ENGINEERS. (2010). ASCE/SEI 7-10: Minimum Design Loads for Buildings and Other Structures. Washington, DC, 2010.

ASSOCIAÇÃO BRASILEIRA DE NORMAS TÉCNICAS. (2008). NBR 8800: Projeto de estruturas de aço e de estruturas mistas de aço e concreto de edifícios. Rio de Janeiro, 2010.

ASSOCIATION OF IRON AND STEEL ENGINEERS. (2003). Technical report no 13: Guide for the design and construction of mill buildings. Pittsburgh, PA, 2003.

BELLEI, I. H. Edifícios industriais em aço: Projetos e cálculo. 3. ed. São Paulo: PINI, 2000. 489 p.

CANADIAN INSTITUTE OF STEEL CONSTRUCTION. (2012). CISC/ICCA: Guide for the design of crane-supporting steel structures. Markham, ON, 2012.

LEITE, L. R. S. Análise numérica de vigas de rolamento de aço sem contenção lateral entre apoios. 2016. 140p. Dissertação (Mestrado em Engenharia Civil (Estruturas)) - Escola de Engenharia de São Carlos, Universidade de São Paulo, São Carlos, 2017.

MORI, D. D.; NETO, J. M. Flexo-torção: barras de seção delgada aberta. 1 ed. São Carlos: EESCUSP, 2009. $180 \mathrm{p}$. 